\title{
Photoluminescent arginine-functionalized polycitrate with enhanced cell activity and hemocompatibility for live cell bioimaging
}

\author{
Min Wang, ${ }^{1 \#}$ Yi Guo, ${ }^{1 \#}$ Peter X. Ma, ${ }^{2,3}$ Bo Lei ${ }^{1,4,5}$ \\ ${ }^{1}$ Frontier Institute of Science and Technology, State Key Laboratory for Mechanical Behavior of Materials, Xi'an Jiaotong \\ University, Xi'an, 710054, China \\ ${ }^{2}$ Department of Biomedical Engineering, Macromolecular Science and Engineering Center, University of Michigan, Ann Arbor, \\ Michigan, 48109-1078 \\ ${ }^{3}$ Department of Materials Science and Engineering, University of Michigan, Ann Arbor, Michigan, 48109-1078 \\ ${ }^{4}$ State Key Laboratory for Manufacturing Systems Engineering, Xi'an Jiaotong University, Xi'an, 710054, China \\ ${ }^{5}$ Instrument Analysis Center, Xi'an Jiaotong University, Xi'an, 710054, China
}

Received 20 May 2018; revised 26 June 2018; accepted 5 July 2018

Published online 5 October 2018 in Wiley Online Library (wileyonlinelibrary.com). DOI: 10.1002/jbm.a.36512

\begin{abstract}
Development of biodegradable and highly biocompatible polymer with intrinsical photoluminescence and high photostability for real-time live cell bioimaging has attracted much attention recently. Here, a biodegradable and amphiphilic poly (citrate)-co-poly (ethylene glycol) (PEG) grafted with arginine (PCGA) polymer with intrinsical fluorescence was synthesized for targeted live cell bioimaging. The physicochemical structure, photoluminescent properties, hemocompatibility, cytotoxicity, and fluorescent bioimaging studies in live cells were determined in detail. PCGA showed a significantly high hemocompatibility, low cytotoxicity, and excellent photostability, which allows for imaging the live cells attachment and proliferation. Furthermore, PCGA could efficiently enhance cell
\end{abstract}

attachment and proliferation due to the presence of arginine, suggesting their high cellular biocompatibility. Importantly, PCGA could selectively stain the lysosome in cells. Our results demonstrated that the amino acid-based polymer functionalization may be an important strategy to develop multifunctional biomaterials with enhanced biocompatibility for targeted bioimaging, cancer therapy, and regenerative medicine. (c) 2018 Wiley Periodicals, Inc. J Biomed Mater Res Part A: 106A: 31753184, 2018.

Key Words: bioactive biomaterials, poly(citrate), arginine, intrinsical photoluminescence, live cell bioimaging

How to cite this article: Wang M, Guo Y, Ma PX, Lei B. 2018. Photoluminescent arginine-functionalized polycitrate with enhanced cell activity and hemocompatibility for live cell bioimaging. J Biomed Mater Res Part A 2018:106A:3175-3184.

\section{INTRODUCTION}

Live cell imaging is a powerful tool to continuous observe the activities within the live cells and becomes increasingly important for biomedical applications. ${ }^{1,2}$ Specially, by the advanced fluorescent bioimaging techniques, the critical insight of biological activities in subcellular and molecular levels in living cells could be demonstrated., ${ }^{3,4}$ Currently, the most widely used fluorescent probes are still organic dyes such as fluorescein isothiocyanate (FITC) and 5-carboxyfluorescein (Fam), but the main limitations in conventional fluorescence dyes include poor hydrophilicity, low photostability, and instability in bio-environment. ${ }^{5}$ Compared with the conventional fluorescence dyes, many novel biomaterials have been explored for bioimaging applications because of their highly photostability such as inorganic fluorescent materials and aggregation-induced emission (AIE) polymers and so on. ${ }^{6-9}$ However, most of current organic and inorganic fluorescent materials are still not biodegradable and may have potential toxicity concerns in large-scale applications. Therefore, it is very urgent and necessary to develop highly biocompatible and biodegradable biomaterials with intrinsical stable photoluminescence for live cell imaging applications.

In recent years, poly (citrate) (PC)-based polymers have been gaining great interest in biomedical application such as tissue regeneration and gene delivery, due to their high biocompatibility, controlled biodegradation, and low cost. ${ }^{10,11}$ In previous study, our group developed a series of highly

\#These authors contributed equally to this work.

Correspondence to: Bo Lei; e-mail: rayboo@xjtu.edu.cn

Contract grant sponsor: State Key Laboratory for Mechanical Behavior of Materials; contract grant number: 20161801

Contract grant sponsor: China Postdoctoral Science Foundation; contract grant number: 2017M613148

Contract grant sponsor: National Natural Science Foundation of China; contract grant number: 51502237 and 51502237 and 51872224 and U1501245 
elastomeric PC-based hybrid polymers, demonstrated their potential biomedical applications including bone tissue repair and gene delivery in vitro and in vivo. ${ }^{12-14}$ However, the conventional PC polymer shows the poor hydrophilicity and photoluminescent property, which limit their promising application in live cell imaging. On the other hand, the cellular biocompatibility of PC-based polymers is also needed to be improved for their long-term biomedical applications.

Polyethylene glycol (PEG), as a biocompatible polymer, has been widely used to modify hydrophobic polymers to improve their solubility. ${ }^{15,16}$ As a semiessential amino acid in human, L-arginine plays an important role in biological activity such as cell division and wounds healing. ${ }^{17}$ Therefore, the functionalization of L-arginine on PC-based polymers may significantly enhance their biocompatibility. In addition, amine-abounded branched polymers such as the hyperbranched poly(amine-ester), poly(propyletherimine) dendrimers, and poly(amidoamine) present significant inherent photoluminescent properties. ${ }^{18}$ As an alkaline amino acid, L-arginine functionalized PC-based polymer may also exhibit stable photoluminescent ability. Conventional polymers with intrinsic photoluminescent properties are usually conjugated polymers containing conjugated main chain or $\pi$-aromatic building blocks. ${ }^{19}$ However, conjugated polymers are always hydrophobic, non-biodegradable, multistep processing, which limit their wide biomedical applications such as long-term live bioimaging and tissue engineering. Therefore, the development of nonconjugated photoluminescent polymer with control biodegradation and biocompatibility is very necessary in biomedical applications.

In this study, we report the synthesis of water-soluble PC-co-PEG grafted with L-arginine (PCGA), and investigate the effect of L-arginine on the photoluminescent properties, blood compatibility, and cellular compatibility. We also demonstrate the live cell imaging application of PCGA.

\section{MATERIALS AND METHODS}

\section{Materials}

Citric acid (99\%), 1,8-octanediol (OD) (98\%), PEG (1 kDa), and 4-(2-hydroxyethyl)-1-piperazineethanesulfonic acid (HEPES) were obtained from Sigma-Aldrich. 1-(3-Dimethylaminopropyl)3-ethylcarbodiimide hydrochloride (EDC, 99\%), N-hydroxy succinimide (NHS, 98\%) and 2-(N-Morpholino) ethanesulfonic acid (MES, 99\%) were purchased from J\&K Scientific. Phosphate buffered saline (PBS), Dulbecco's Modified Eagle Medium (DMEM), 4'-6-diamidino-2-phenylindole (DAPI), LIVE/DEAD staining kit, Alamar Blue kit, Mito-Tracker Green and LysoTracker Red were bought from Invitrogen. All chemicals were used as received.

\section{Synthesis of PCGA polymer}

The PCGA polymer was synthesized via a typical catalytic reaction of EDC and NHS using PCG and arginine in $50 \mathrm{mM}$ MES buffer anhydrous, and the PCG prepolymer was synthesized according to our previous report. ${ }^{20}$ To synthesize the PCGA polymer, the PCG prepolymer was first added into MES buffer anhydrous include EDC and NHS to activate the carboxyl groups. After $30 \mathrm{~min}$, arginine with various amounts was added in the reaction system, and the reaction mixtures were continuously stirred for $24 \mathrm{~h}$ at room temperature. Finally, the resulted PCGA polymer was purified by dialysis using a dialysis tube (MWCO 1000) for 3 days. The final PCGA polymers were collected after freeze-drying and stored until use.

\section{Physicochemical structure characterization of PCGA polymers}

The ${ }^{1} \mathrm{H}$ nuclear magnetic resonance $\left({ }^{1} \mathrm{H}\right.$ NMR) instrument (Ascend $400 \mathrm{MHz}$, Bruker) and Fourier transformation infrared (FT-IR) spectroscopy (NICOLET 6700, Thermo) were performed to determine the chemical compositions and structures of PCG and PCGA polymers. Briefly, for ${ }^{1} \mathrm{H}$ NMR analysis, the polymers were dissolved in dimethyl sulfoxided6 (DMSO-d6) with a concentration of $40 \mathrm{mg} / \mathrm{mL}$. The polymers were mixed with $\mathrm{KBr}$ to be pressed into a transparent slice for FT-IR measurement.

\section{Photoluminescence capacity evaluation of PCGA polymers}

The photoluminescent (PL) spectra of PCGA polymers solutions with different concentrations were obtained by a spectrophotometer (F-4500, Hitachi) equipped with a $150 \mathrm{~W}$ Xe arc lamp at room temperature, and the slit widths of excitation and emission were $2.5 \mathrm{~nm}$ for all samples. The fluorescence lifetime and quantum yield of PCGA were obtained by a steady-state and transient fluorescence spectroscopy (FLS980, Edinburgh), respectively. The fluorescent images of PCGA polymers solutions were obtained by excitation at $365 \mathrm{~nm}$ using a UV lamp. The photoluminescent stability of PCGA polymers were evaluated through continuous irradiation for a period of time by excitation at $405 \mathrm{~nm}$ using an inverted fluorescent microscope (IX 71, Olympus).

\section{Hemocompatibility analysis of PCGA polymers}

The rats were ordered from animal medical center in Xi'an Jiaotong University, and all procedures were approved by the Animal Care and Use Committee. The hemocompatibility of PCGA polymers were investigated according to the destructed level of erythrocyte after incubated with the polymers for $1 \mathrm{~h}$. Briefly, the blood, collected in heparinizedtubes from rats, was centrifugated at $1000 \mathrm{rpm}$ for $10 \mathrm{~min}$, and the precipitate was washed three times with PBS buffer solution ( $\mathrm{pH}$ 7.4). The precipitate of erythrocyte was resuspended in the wash buffer at $5 \%(\mathrm{v} / \mathrm{v})$ and used within $24 \mathrm{~h}$ after collection. The polymers solutions prepared in the PBS buffer were added to the erythrocyte at different concentrations for $1 \mathrm{~h}$ in a $37^{\circ} \mathrm{C}$ incubator. The release of hemoglobin was determined using a microplate reader (SpectraMax i3, Molecular Devices) at $540 \mathrm{~nm}$. The completeness of erythrocyte was investigated using a fluorescent microscope (BX51, Olympus). The $0.1 \%$ Triton $\mathrm{X}-100$ was used as the complete hemolysis control value (100\%) and PBS buffer was used as the low hemolysis control value $(0 \%)$. Less than $10 \%$ hemolysis was regarded as good blood compatibility in our experiments. 


\section{Cytotoxicity of PCGA polymers}

The cytotoxicity of PCGA polymers against rat-derived myoblast line (C2C12) was investigated using Alamar blue ${ }^{\circledR}$ assay kit and Live/dead staining kit (Thermal Sci.). Briefly, the $\mathrm{C} 2 \mathrm{C} 12$ cells were incubated with a complete Dulbecco's modified Eagles medium (DMEM, Invitrogen) with 10\% (v/v) fetal bovine serum (FBS) at $37^{\circ} \mathrm{C}$ under a humidified atmosphere containing $5 \% \mathrm{CO}_{2}$. After $24 \mathrm{~h}$, the medium was replaced by a mixed DMEM solution including the polymers with different concentrations $(25 \mu \mathrm{g} / \mathrm{mL}, 50 \mu \mathrm{g} / \mathrm{mL}$, $100 \mu \mathrm{g} / \mathrm{mL}$, and $200 \mu \mathrm{g} / \mathrm{mL}$ ). The cell viability was investigated using a microplate reader (SpectraMax i3, Molecular Devices) through measuring the fluorescent intensity at an excitation/emission wavelength of $530 \mathrm{~nm} / 600 \mathrm{~nm}$ after cultured for $1 \mathrm{~d}$ and $3 \mathrm{~d}$, respectively. Live/dead staining study was also used to investigate cell viability using a fluorescent microscope (BX51, Olympus) to observe the live cells morphology after $\mathrm{d}$ days culture. The tissue culture plate (TCP) without adding any polymer was used as the control.

\section{Fluorescent imaging studies of PCGA polymers}

The fluorescent imaging studies of PCGA polymers $(100 \mu \mathrm{g} / \mathrm{mL})$ in C2C12 cells in vitro were determined using a laser confocal fluorescent microscope (CLSM, TCS SP5, Leica). Briefly, the cells were seeded onto glass cover slips in 24-well plates with a density of $5 \times 10^{4}$ cells per well. After the cells were cultured for $24 \mathrm{~h}$, the slips were treated with medium including PCGA polymers for another $24 \mathrm{~h}$. Subsequently, the slips were washed by PBS three times, fixed by methyl alcohol and acetic acid for $30 \mathrm{~min}$ and stained by FITC for $20 \mathrm{~min}$. Finally, the cellular imaging was observed using a CLSM to determine the fluorescent imaging studies of PCGA polymers. To investigate the fluorescent imaging stability of PCGA polymers in vitro, the cellular imaging was determined by incubating cells with polymers for 1 day and 3-day, and incubating cells through continuous irradiation for different time by excitation at $405 \mathrm{~nm}$.

\section{Mitochondria and lysosome costaining evaluation}

To study the intracellular trafficking, PCGA solution with a concentration of $100 \mu \mathrm{g} / \mathrm{mL}$ was incubated with cells for $30 \mathrm{~min}$, then the PCGA solution was replaced by fresh medium. The cells were co-stained by the Mito-Tracker Green and Lyso-Tracker Red, respectively. Here, the cells were stained as blue by PCGA, green by Mito-Tracker and red by Lyso-Tracker.

\section{Statistical analysis}

All date was expressed as the mean \pm standard deviation (SD) and the experiment were repeated at least three times. Statistical results were determined by a student's $t$-test. The statistically significant difference between groups were considered when $* p<0.05, * * p<0.01$.

\section{RESULTS AND DISCUSSION}

\section{Synthesis and characterizations of PCGA polymers}

The PCGA polymers were synthesized from various monomers via a two steps method. Firstly, the PCG polymer was synthesized using citric acid (CA), 1,8-OD and PEG by a melt polymerization [Fig. 1(A)]. The PCGA polymers were fabricated by further crosslinking L-arginine with PCG through a catalytic reaction of EDC and NHS in anhydrous MES buffer solution [Fig. 1(B)]. In the reaction process of the polymerization, the main chain of polymer was composed of citric acid, 1,8-OD and PEG, and L-arginine were grafted onto the residual carboxyls of PCG through its primary amines. In the structure of PCGA polymer, hydrophobic PC provides biodegradability, hydrophilic PEG provided water-solubility, and Larginine was used to increase biocompatibility and enable photoluminescent ability.

The successful synthesis of PCGA polymer was determined by ${ }^{1} \mathrm{H}$ NMR and FT-IR analysis, as shown in Figure 2. The representative methylene peaks $\left(-\mathrm{CH}_{2}{ }^{-}\right)$identified at $1.2 \mathrm{ppm}, 1.5 \mathrm{ppm}, 3.9 \mathrm{ppm}$, and $4.1 \mathrm{ppm}$ were assigned to 1,8-OD [Fig. 2(A)]. The multiple peaks between $2.6 \mathrm{ppm}$ and $3.0 \mathrm{ppm}\left(-\mathrm{CH}_{2}-\right)$ were attributed to citric acid [Fig. 2(A)]. The peaks at $3.5 \mathrm{ppm}, 4.2 \mathrm{ppm}$, and $4.3 \mathrm{ppm}$ were identified

(A)

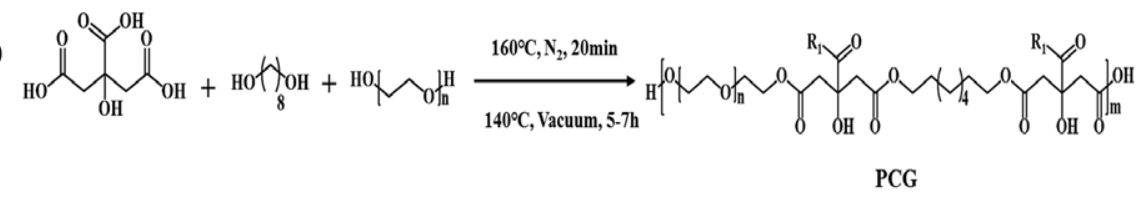

(B)
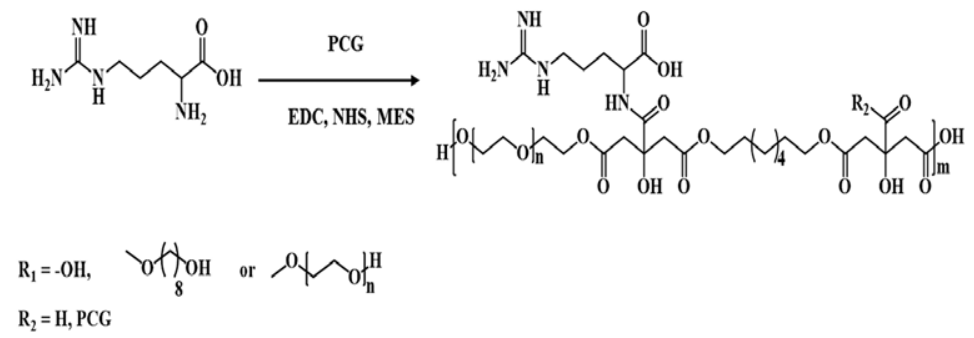

FIGURE 1. Schematic illustrations for synthesizing PCG and PCGA polymers. (A) Polymerization routine of PCG prepolymer; (B) Polymerization process of PCGA polymer through a representative EDC/NHS reaction. 
(A)
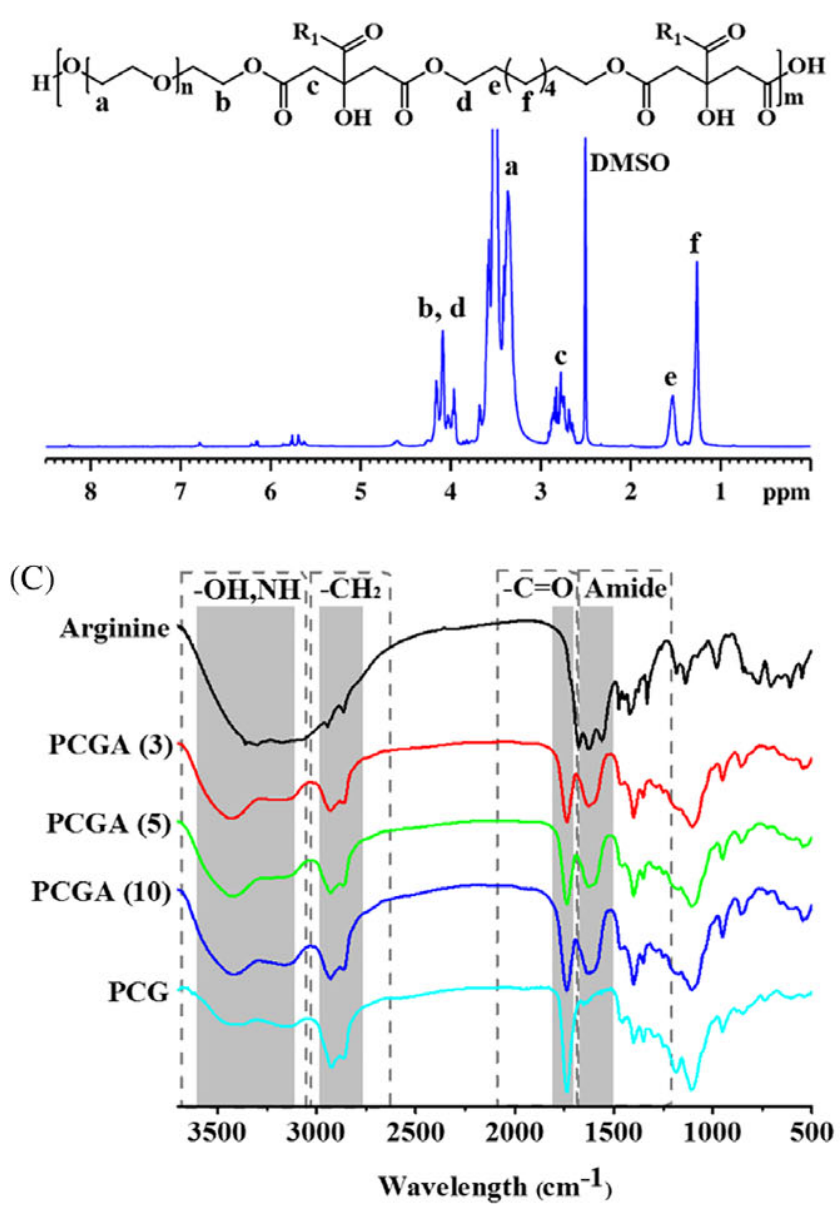

(B)

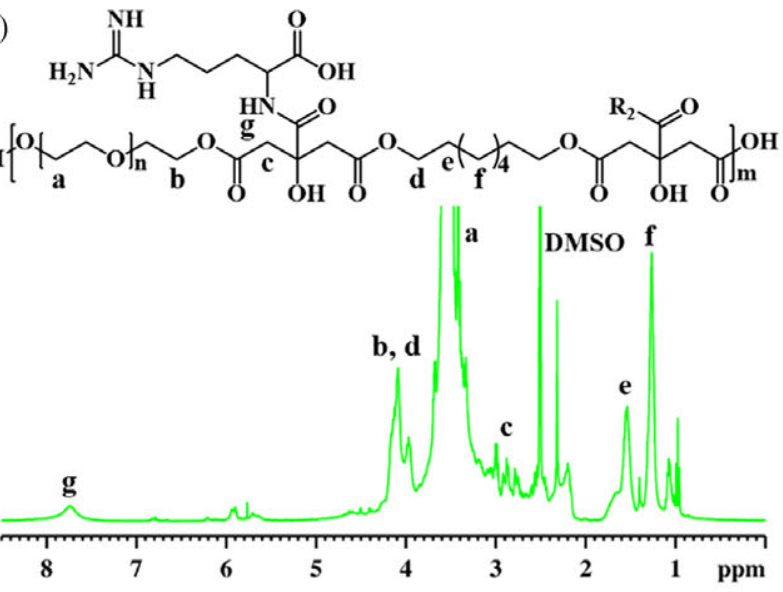

(D)

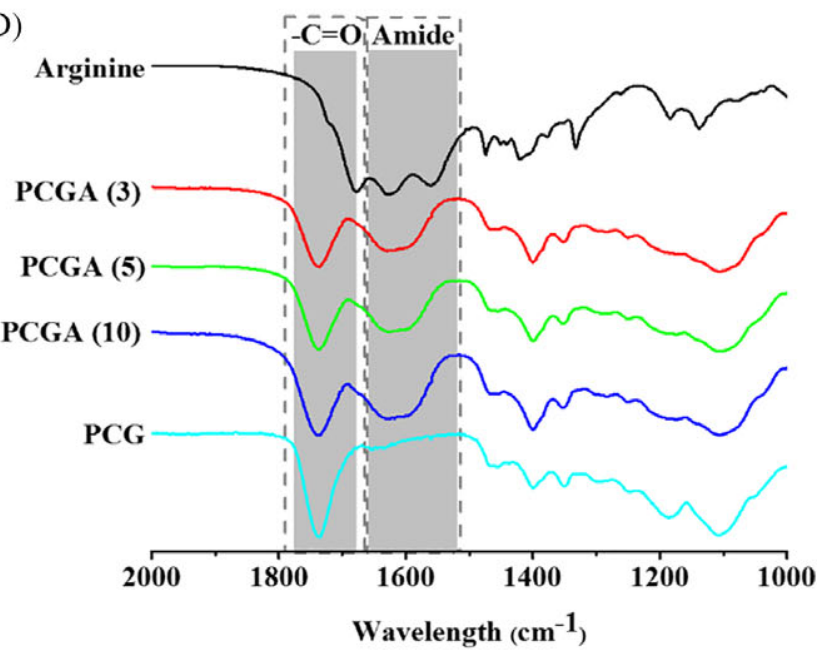

FIGURE 2. Chemical structure characterization of PCG and PCGA polymers. $(A, B){ }^{1} H$ NMR spectra of PCG prepolymer (A) and PCGA polymer (B); (C, D) FT-IR spectra of arginine, PCG and PCGA polymers between $3800-500 \mathrm{~cm}^{-1}$ (C) and $2000-1000 \mathrm{~cm}^{-1}$ (D).

as the methylene $\left(-\mathrm{CH}_{2}-\right)$ of PEG. The presence of peaks at $3.9 \mathrm{ppm}, 4.1 \mathrm{ppm}, 4 . \overline{\mathrm{ppm}}, 4.3 \mathrm{ppm}\left(-\mathrm{CH}_{2}-\right)$ indicated the successful synthesis of PCG [Fig. 2(A)]. The presence of characteristic peaks at $7.8 \mathrm{ppm}(-\mathrm{NH}-)$ confirmed the successful grafting of arginine into the PCG prepolymer [Fig. 2(B)]. In addition, the molar weight ratios of the monomer (CA:OD: PEG:Arg) was 1:0.72:0.28:0.22 by the analysis ${ }^{1} \mathrm{H}$ NMR spectra. The further analysis demonstrated that the actual arginine grafting ratio in PCG was about The presence of characteristic peaks assigned to $-\mathrm{OH}$ between $3300 \mathrm{~cm}^{-1}$ and $3700 \mathrm{~cm}^{-1},-\mathrm{CH}_{2}$ - between $2800 \mathrm{~cm}^{-1}$ and $3000 \mathrm{~cm}^{-1}$, $-\mathrm{C}=0$ at $1747 \mathrm{~cm}^{-1}$ and $-\mathrm{C}(=0) \mathrm{NH}-$ at $1620 \mathrm{~cm}^{-1}$ were confirmed by FT-IR spectra [Fig. 2(C,D)]. ${ }^{1} \mathrm{H}$ NMR and FT-IR analysis indicated the successful synthesis of PCG and PCGA polymers.

\section{Photoluminescence ability of PCGA polymers}

The inherent photoluminescent properties of PCGA polymers are shown in Figure 3. Compared with the PCG prepolymer, the PCGA polymers exhibited an optimum excitation wavelength of $395 \mathrm{~nm}$ [Fig. 3(A)]. The stronger fluorescent emission at $490 \mathrm{~nm}$ was found from PCGA and no significant fluorescent emission was observed in PCG polymer [Fig. 3(B)]. The fluorescent emission (490 nm) of PCGA was significantly increased with the polymer concentration, suggesting the ignorable aggregation-induced fluorescent quenching effect which was usually presented in organic dyes [Fig. 3(C)]. ${ }^{21}$ Additionally, under different excitation wavelength, the emission wavelength of PCGA showed a slightly red shift to be $520 \mathrm{~nm}$ [Fig. 3(D)]. It should be indicated that PCGA also exhibited high fluorescent stability against acid/alkaline and temperatures [Fig. 3(E,F)]. The fluorescent emission spectra of PCGA solution just presented a little change at the intensity between $\mathrm{pH} 3.9$ to $\mathrm{pH} 8.3$ and $25^{\circ} \mathrm{C}$ to $50^{\circ} \mathrm{C}$, suggesting their high photoluminescent stability at various environments. There were no significant differences in fluorescent emission position of PCGA polymer as different $\mathrm{pH}$ values and temperatures. Furthermore, as compared to fluorescein isothiocyanate (FITC), the fluorescence of PCGA polymers was still strong under continuously irradiation for $10 \mathrm{~min}$ at the excitation of $405 \mathrm{~nm}$, but the intensity of FITC has been decreased 
(A)

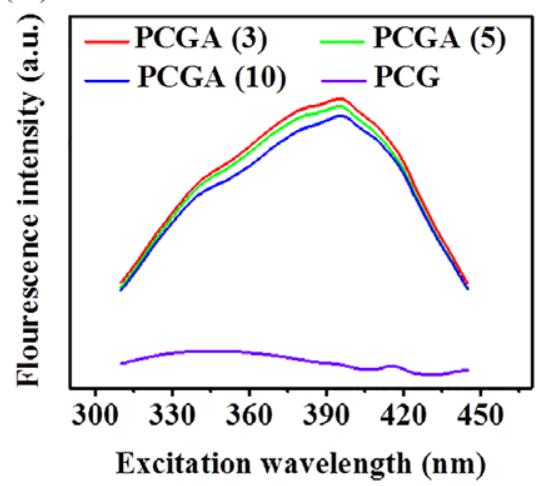

(D)

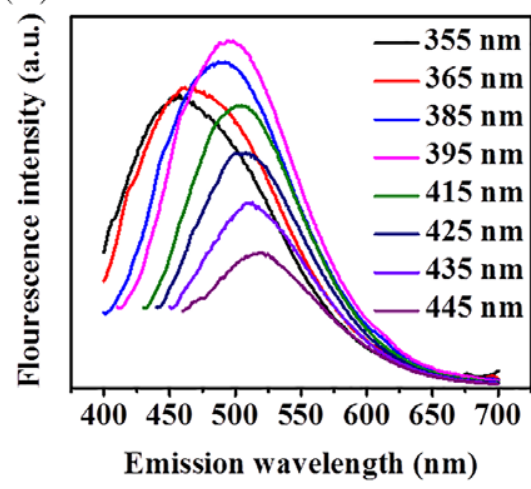

(G)

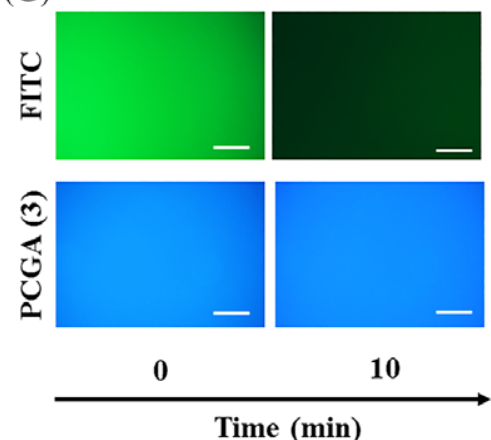

(B)

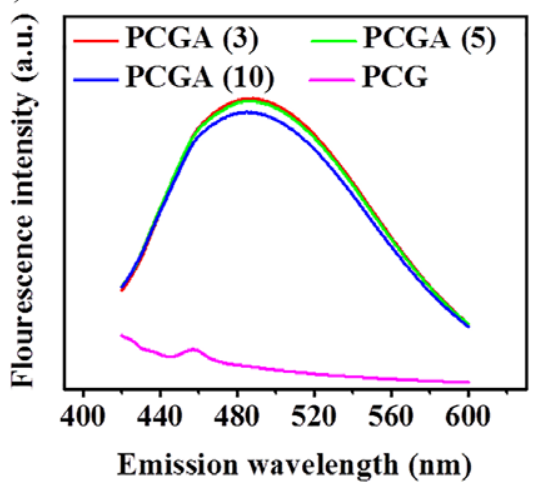

(E)

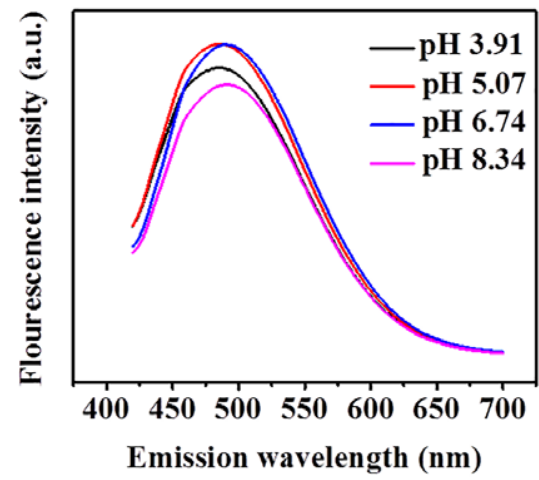

(H)

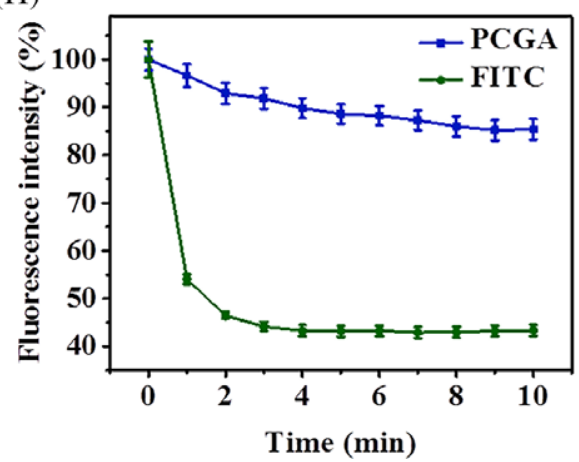

(C)

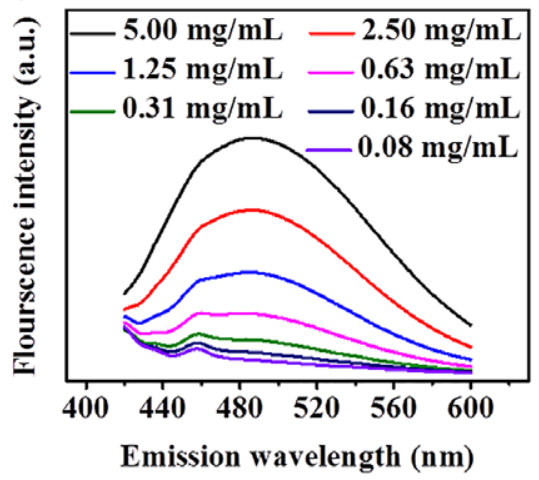

(F)

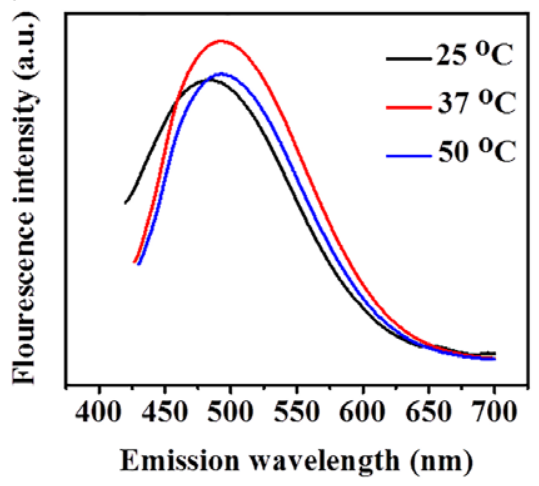

(I)

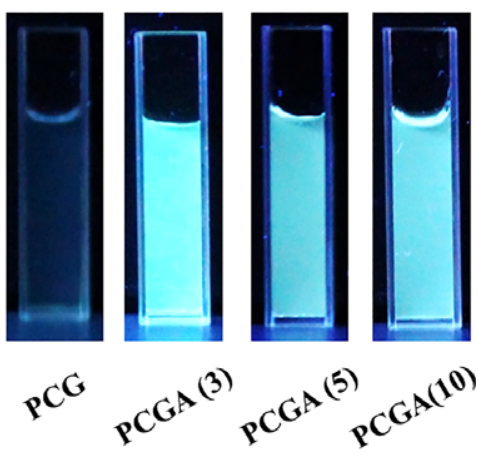

FIGURE 3. Intrinsically photoluminescent ability of PCGA polymers. (A) Excitation spectra of PCG and PCGA polymers in deionized water under the emission wavelength of $490 \mathrm{~nm}$; (B) Emission spectra of PCG and PCGA polymers in deionized water under the excitation wavelength of $395 \mathrm{~nm}$; (C-F) Emission spectra of PCGA (3) polymer in deionized water under different concentrations (C), excitation wavelengths (D), pH values (E), temperatures (F); (G) Fluorescent images of PCGA (3) polymer irradiated for different time intervals by an inverted fluorescent microscope under $405 \mathrm{~nm}$ excitation (scale bar:100 $\mu \mathrm{m}$ ); (H) Fluorescent stability after irradiated for various times by a high pressure mercury lamp (365 $\mathrm{nm}$ ); (I) Fluorescent images of PCG and PCGA polymers in deionized water $(1.25 \mathrm{mg} / \mathrm{mL})$ under $365 \mathrm{~nm}$ excitation by a UV lamp.

significantly [Fig. 3(G,H)]. The stable and bright blue light in PCGA solution with different arginine contents could be observed under a UV lamp at the excitation of $365 \mathrm{~nm}$ [Fig. 3(I)]. These results demonstrated that PCGA polymers possessed a highly stable fluorescent emission under various conditions including acid-alkaline environment, temperature, and long-time light irradiation. In addition, the fluorescence lifetime and fluorescence quantum yield of PCGA were 3.03 ns and $11.00 \%$, respectively. In recent years, it was shown that some nonconjugated polymers containing amine group such as poly(amidoamine) showed excellent fluorescent emission. ${ }^{22,23}$ Further studies exhibited that the heteroatom-containing double bonds in these nonconjugated polymers may contribute to their excellent photoluminescent performance. $^{24}$ The detailed fluorescent mechanism of amine-contained nonconjugated polymers is still under investigation. In PCGA, there were many heteroatom-containing double bonds such as $\mathrm{C}=\mathrm{N}$ and $\mathrm{C}=\mathrm{O}$ which may arouse the strong photoluminescence. The high photoluminescent ability and photostability of PCGA make them highly competitive for live cell bioimaging. 


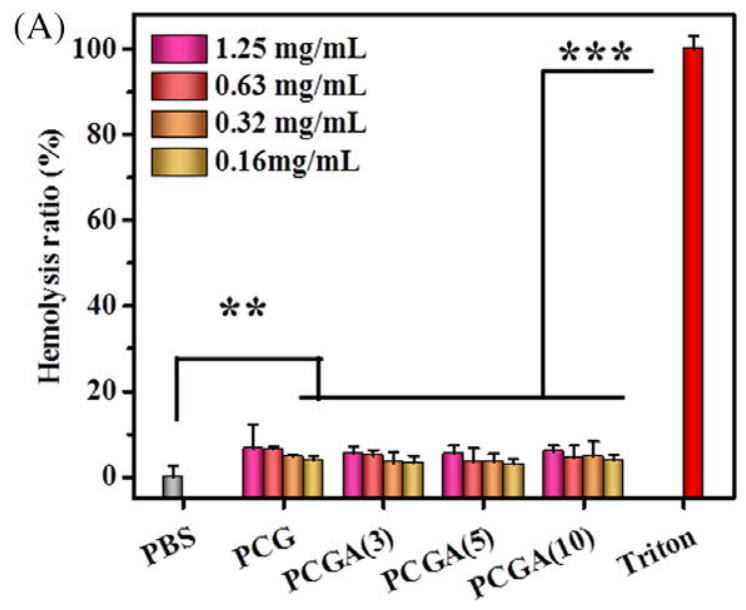

(B)
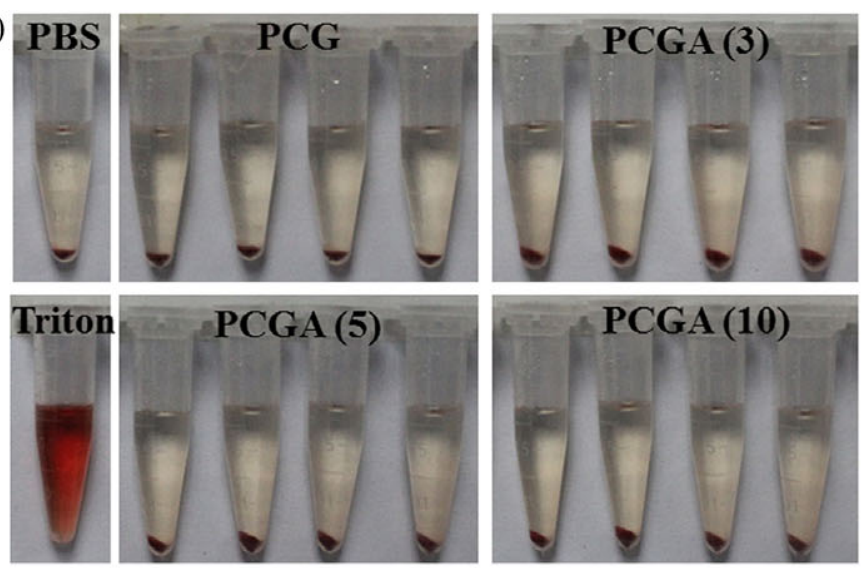

$\begin{array}{llllllllll}1.25 & 0.63 & 0.32 & 0.16 & 1.25 & 0.63 & 0.32 & 0.16\end{array}$

Concentration $(\mathrm{mg} / \mathrm{mL})$

(C)
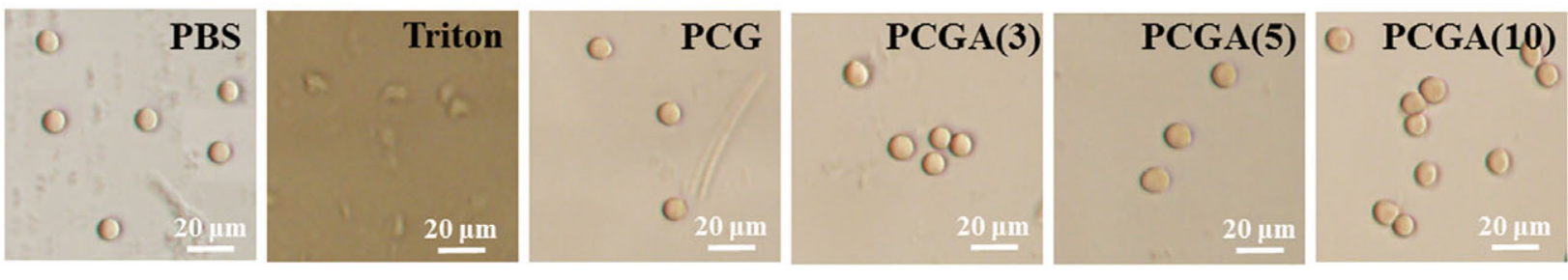

FIGURE 4. Hemocompatibility evaluation of PCGA polymers. (A) Hemolysis ratio of rat erythrocyte after $1 \mathrm{~h}$ incubation with different concentrations of various polymers at $37^{\circ} \mathrm{C}$; (B) Photos of hemolysis of erythrocyte after $1 \mathrm{~h}$ incubation with different polymers at various concentrations at $37^{\circ} \mathrm{C}$; (C) Morphology change of rat erythrocyte after $1 \mathrm{~h}$ incubation with various polymers $(1.25 \mathrm{mg} / \mathrm{mL})$ at $37^{\circ} \mathrm{C}$. Triton and PBS were used as positive and negative control, respectively $(* * p<0.01, * * * p<0.001)$.

\section{Hemocompatibility and cellular biocompatibility evaluations of PCGA polymers}

The hemocompatibility of PCGA polymers were investigated through analyzing the release of hemoglobin (Fig. 4). After $1 \mathrm{~h}$ incubation with different polymer concentrations in the range of $0.16-1.25 \mathrm{mg} / \mathrm{mL}$ at $37^{\circ} \mathrm{C}$, PCG, PCGA (3), PCGA (5), PCGA (10) showed significantly low hemolytic effects up to $1.25 \mathrm{mg} / \mathrm{mL}$ compared with the Triton and PBS [Fig. 4(A,B)]. The further analysis indicated that the morphology of erythrocyte was not damaged after incubation with various polymers [Fig. 4(C)]. These results demonstrated that PCGA polymer possessed good hemocompatibility and may be promising for further biomedical applications. ${ }^{25}$

The cytotoxicity of PCGA polymers were evaluated in C2C12 cells using Alamar blue ${ }^{\circledR}$ assay and live/dead staining study (Fig. 5). The PCG and PCGA polymer did not exhibit any significant cytotoxicity at different concentrations $(25 \mu \mathrm{g} / \mathrm{mL}, 50 \mu \mathrm{g} / \mathrm{mL}, 100 \mu \mathrm{g} / \mathrm{mL}$, and $200 \mu \mathrm{g} / \mathrm{mL})$ against $\mathrm{C} 2 \mathrm{C} 12$ cells after cultured for $72 \mathrm{~h}$, indicating their good cytocompatibility (Fig. 5). Interestingly, C2C12 cells showed significantly high proliferation after incubated with PCGA polymers for 1 and 3 day in all range of concentration, as compared to TCP and PCG [Fig. 5(A,B)]. Meanwhile, the Live/dead staining study showed the good cell attachment and cell numbers on PCGA polymer compared with the PCG polymers [Fig. 5(C,D)]. The reason may be that the trace amount of positive charge of polymers influenced the cell fate due to the introduction of L-arginine in PCGA. Larginine have been investigated that it can facilitate the cellular uptake of covalently conjugated particles due to the existence of guanidyl group, and may improve cellular proliferation by increasing specific receptor expression and the utilization of interleukin-2. ${ }^{26-28}$ These results demonstrated that the addition of L-arginine could efficiently enhance the cellular biocompatibility of PCG polymer.

\section{Live cell imaging studies of PCGA polymers}

Based on the excellent multifunctional properties of PCGA polymers such as highly stable photoluminescence and enhanced cellular biocompatibility, the in vitro live cell imaging applications were investigated by CLSM. The live cell imaging, proliferation tracking and photostability of PCGA were observed (Fig. 6). After incubated with PCGA at the concentration of $100 \mu \mathrm{g} / \mathrm{mL}$, the cells were stained as blue clearly [Fig. 6(A)]. The cell morphology and nucleus was also stained as green and blue by commercial FTIC [Fig. 6(A)]. After mergence, completed cell morphology could be imaged by different fluorescent molecules.

Significantly, the fluorescence of POCG was still observed clearly after incubated with C2C12 cells for 3 days. Additionally, compared with day 1 , the cell numbers were increased significantly at day 3 , suggesting potential long-term live cell imaging of PCGA [Fig. 6(B,C)]. To show the advantage of PCGA, the photostability in cell imaging was observed 

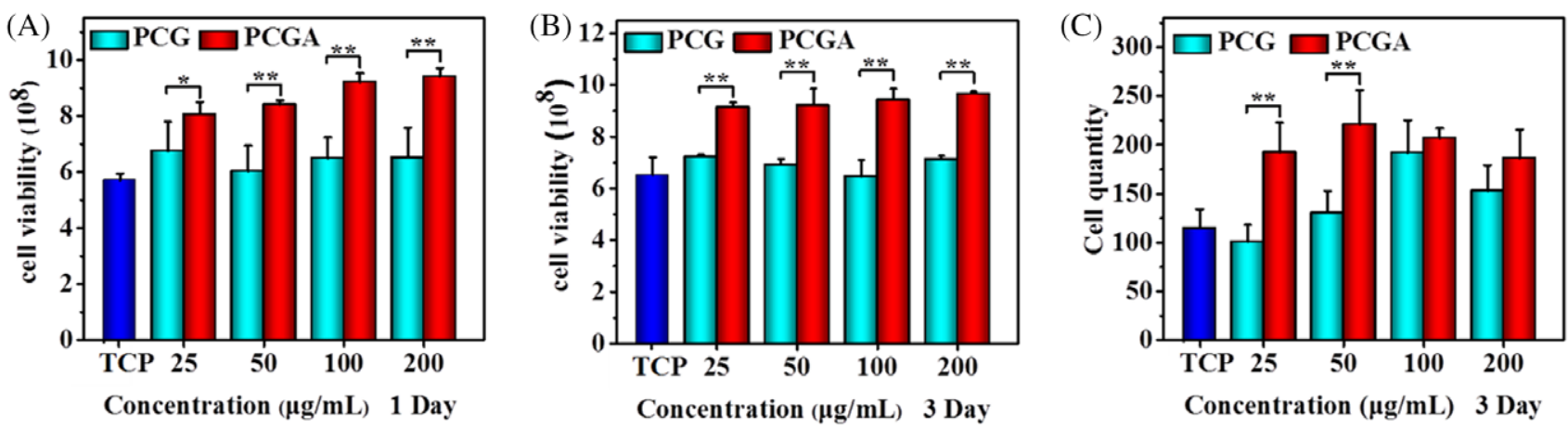

(D)
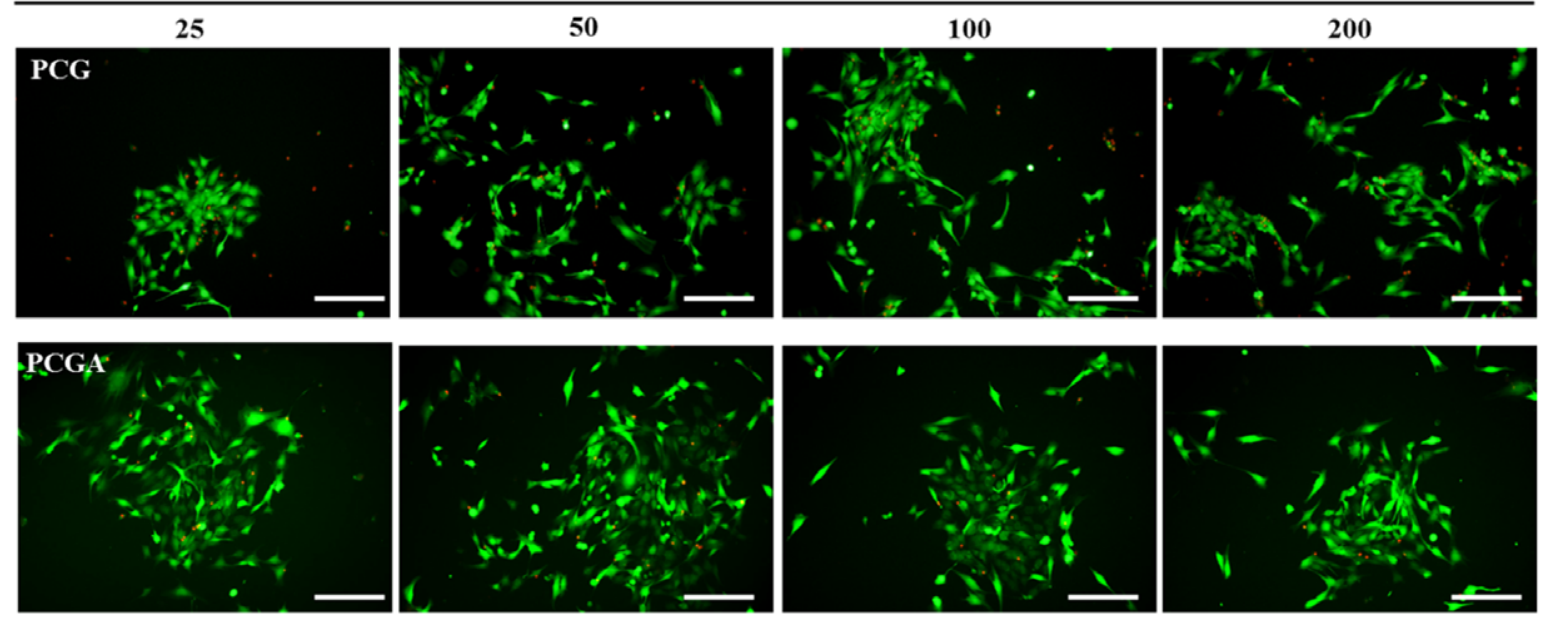

FIGURE 5. Cytocompatibility analysis of PCG and PCGA polymers. (A, B) C2C12 cells viability after incubation with PCG and PCGA polymers for 1 and 3 days at different concentrations $(* p<0.05, * * p<0.01)$; (C) Live C2C12 cells numbers after culture with PCGA at day 3 ; (D) Live C2C12 morphology after 3 days incubation with PCG and PCGA at different concentrations (scale bars: $200 \mu \mathrm{m}$ ).

through continuous irradiation by CLSM, as compared to commercial FITC [Fig. 6(D)]. After $10 \mathrm{~min}$ irradiation, the blue fluorescence of PCGA was still seen clearly, however, the fluorescence of FITC-labeled cells was significantly decreased due to the well-known photobleaching [Fig. 6(D, E)]. These results demonstrated that PCGA polymer could efficiently image the live cells, tracking their proliferation with high photostability.

\section{Intracellular mitochondria and lysosome staining}

To investigate the selective distribution and imaging of PCGA, the mitochondria and lysosome costaining with PCGA in cells were performed. The mitochondria and lysosome in cells was labeled by a commercial Mito-Tracker (Green) and Lyso-Tracker (Red), respectively. As shown in Figure 7, the cell was stained as blue by PCGA and green by Mito-Tracker. After merging PCGA and Mito-Tracker Green, just part of the overlap was observed, indicating the low mitochondria selectivity of PCGA. Then the lysosome was also costained with Lyso-Tracker Red and PCGA. Different with mitochondria staining, the Lyso-Tracker staining (red) was well overlapped with PCGA (blue). This result suggested that PCGA could selectively stain the lysosome in cells. It is well known that lysosome possesses a representative acidic microenvironment. ${ }^{29}$ In this study, PCGA shows an alkaline structure (arginine) which may have strong interaction with lysosome and then presents the selective staining with lysosome (Fig. 7).

In this study, we developed a biodegradable and nonconjugated amino acid-based soluble polycitrate polymer with highly stable photoluminescent capacity and excellent biocompatibility for lysosome-selective live cell imaging. As compared to conventional photoluminescent polymers and inorganic nanoparticles used in bioimaging, as-developed PCGA polymers possess several advantages. ${ }^{30-33}$ Firstly, PCGA is biodegradable amphiphilic polyester which was composed of citric acid, PEG, and arginine, and the introduction of PEG greatly improved the hydrophilicity of the polymer. Therefore, the degradable monomers are highly biocompatible and inexpensive, which enables their large-scale fabrication and applications. Secondly, PCGA could efficiently stain the lysosome in cells with high fluorescent stability, which provides a promising application in targeted bioimaging and cancer therapy. Thirdly, the introduction of L-arginine greatly improves the biocompatibility of the polymer, which could efficiently promote cell proliferation. Finally, PCGA possess many free carboxyls and hydroxyls in their side chains, which makes them facile functionalization for enhancing their biomedical 
(A)
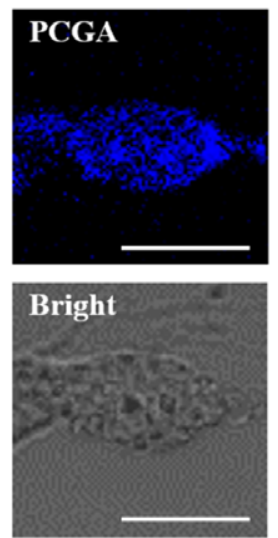

(B)
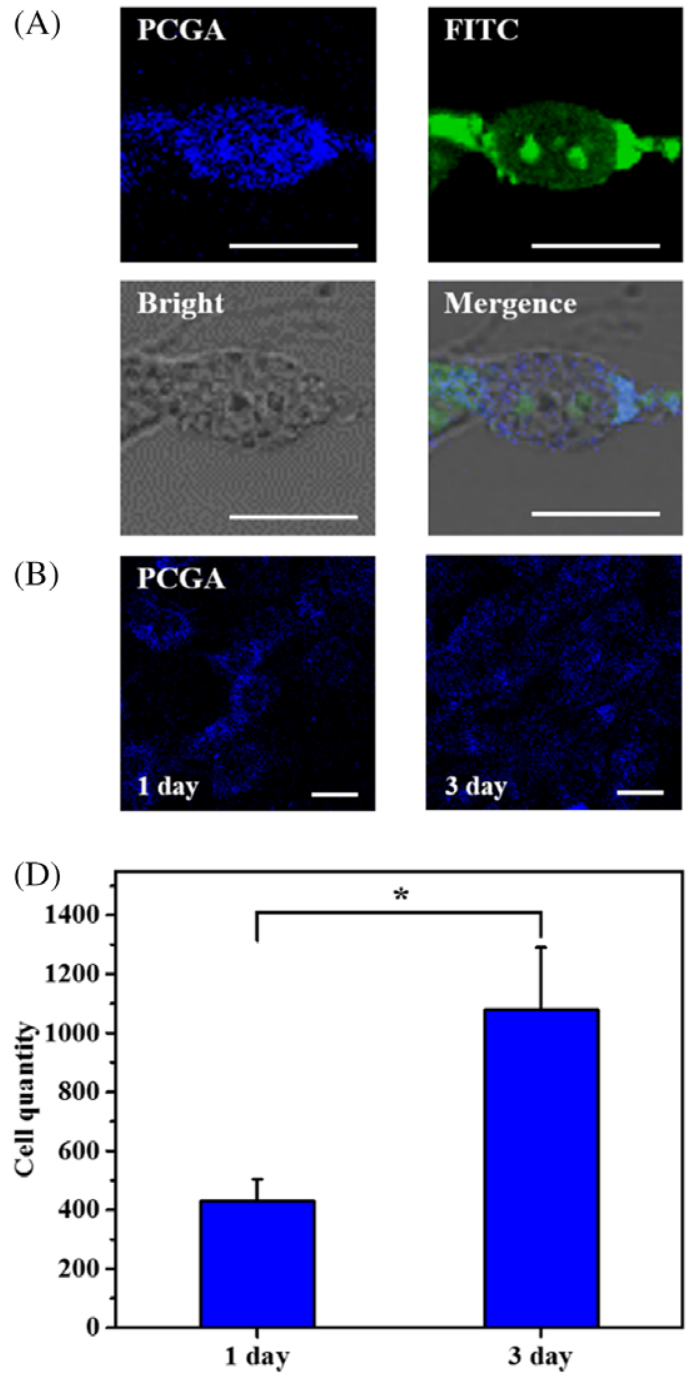

(C)

FICC
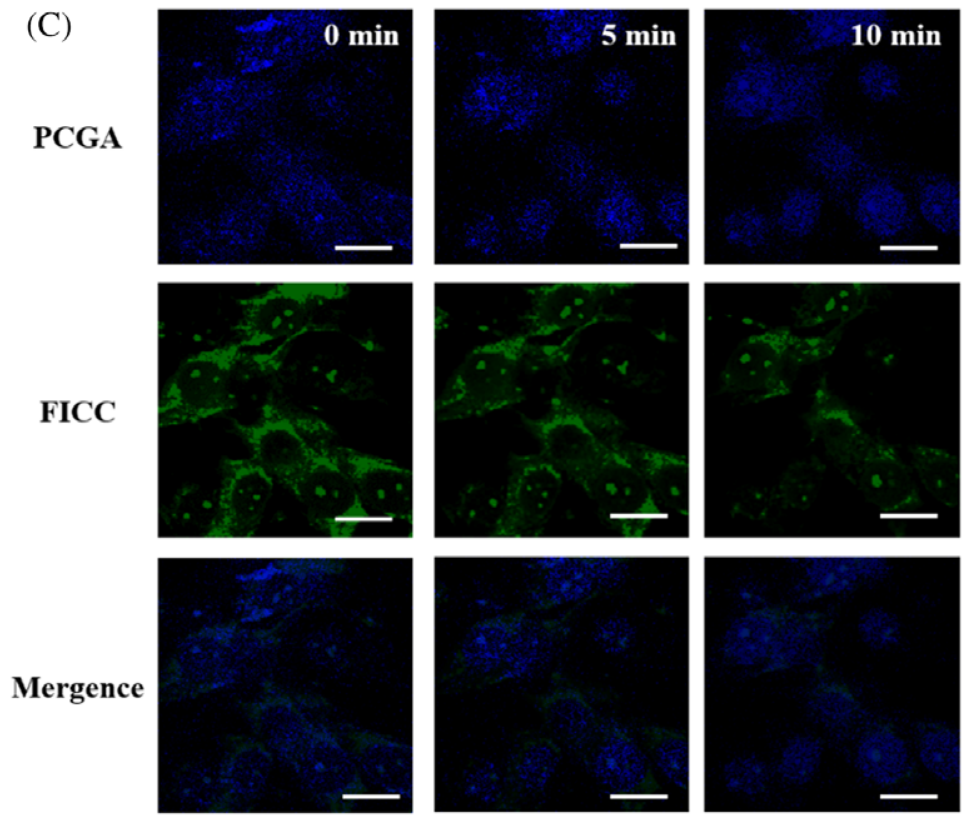

(E)

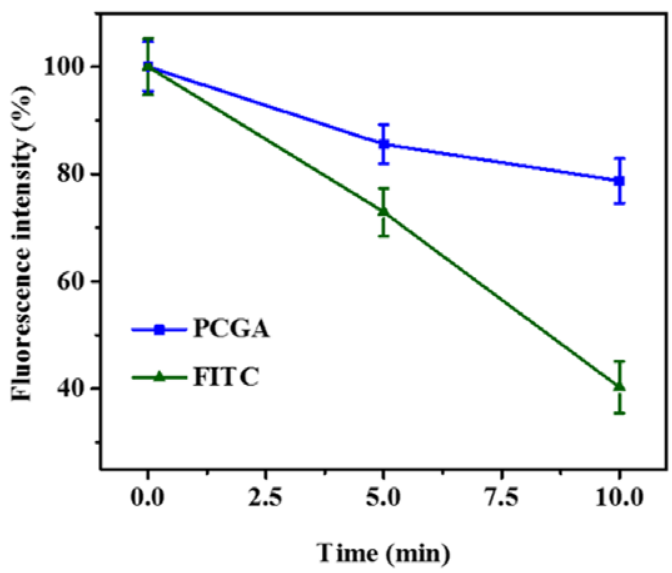

FIGURE 6. Live cells bioimaging evaluation of PCGA polymer in vitro (scale bars $=20 \mu \mathrm{m}$ ). (A) Fluorescent images of C2C12 cells after incubated with PCGA polymer for $24 \mathrm{~h}$ (PCGA: blue, FITC: green, scale bar: $50 \mu \mathrm{m}$ ); (B) Fluorescence images of C2C12 cells after incubated with PCGA for 1 and 3 days, showing the proliferative cell imaging (scale bar: $100 \mu \mathrm{m}$ ); (C) Live cell numbers stained by PCGA after culture for 1 and 3 days $(* * p<0.01)$; (D) Fluorescence stability evaluation through tracking the cellular imaging after continuous irradiation for different time, using commercial FITC as control (cells was incubated with PCGA for $24 \mathrm{~h}$, scale bar: $80 \mu \mathrm{m}$ ); (E) Relative fluorescent intensity of C2C12 after continued irradiation for various times $(* p<0.05, * * p<0.01)$. The PCGA stained cells were excited at $405 \mathrm{~nm}$, FITC stained cells were excited at $488 \mathrm{~nm}$.

applications. For example, PCGA can be grafted using various fluorescent molecules to extend their emission up to near infrared region (NIR, 650-900 nm) which was considered to be an optimum window for in vivo bioimaging. ${ }^{34}$ For targeted imaging and therapy, PCGA can also be functionalized by targeted peptide or molecules. For long-term imaging and cancer therapy, the further bio-functionalization and the in vivo performance should be carried out.

\section{CONCLUSIONS}

In summary, novel biodegradable amino acid-functionalized nonconjugated polycitrate polymers with strong and stable intrinsical fluorescence were successfully synthesized using biocompatible citric acid, PEG, and L-arginine. PCGA exhibited highly stable photoluminescence. Importantly, PCGA polymer showed a good hemocompatibility, significantly enhanced cellular biocompatibility in which cell viability was significantly improved. The further cellular imaging results showed that PCGA could efficiently image live cell and track cell proliferation with high fluorescent stability. PCGA could also selectively stain the lysosome in cells, demonstrating their potential targeted bioimaging and cancer therapy applications. The facile synthesis and functionalization, strong photoluminescence, high biocompatibility and excellent lysosome-selective live cell imaging performance make PCGA highly promising as novel biodegradable water-soluble biomaterials for targeted live cell bioimaging and potential cancer therapy. 


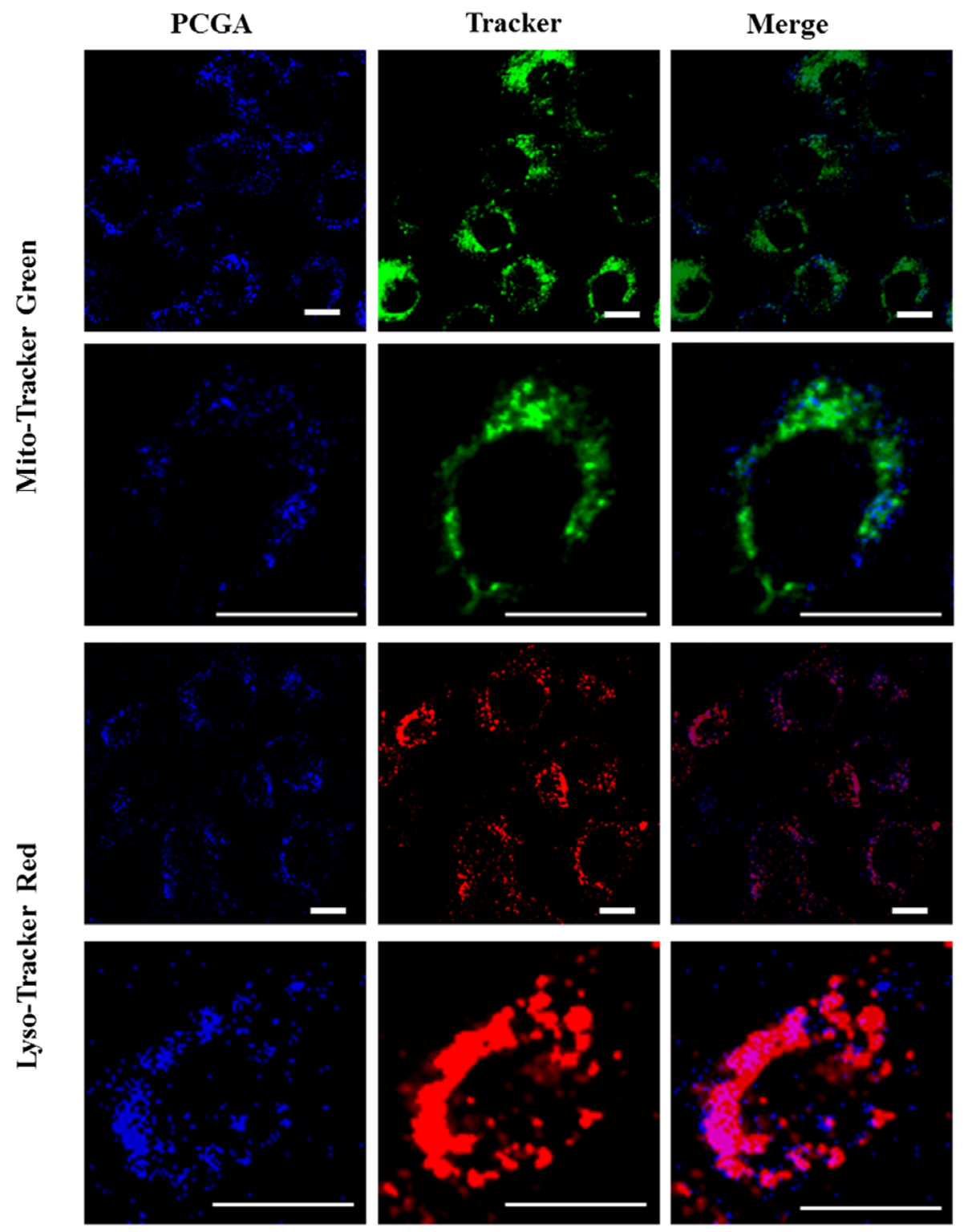

FIGURE 7. Intracellular organelles staining analysis of PCGA using Mito-Tracker Green and Lyso-Tracker Red as the control (scale bars $=20 \mu \mathrm{m}$ ). The staining position of PCGA was well matched with that of Lyso-Tracker, suggesting that PCGA could selectively image the lysosome in cells.

\section{ACKNOWLEDGMENTS}

The authors acknowledge the valuable comments of potential reviewers.

\section{REFERENCES}

1. Oin W, Li K, Feng G, Li M, Yang Z, Liu B, Tang B. Bright and photostable organic fluorescent dots with aggregation-induced emission characteristics for noninvasive long-term cell imaging. Adv Funct Mater 2014;24:635-643.

2. Xue Y, Du Y, Yan J, Liu Z, Ma PX, Chen X, Lei B. Monodisperse photoluminescent and highly biocompatible bioactive glass nanoparticles for controlled drug delivery and cell imaging. J Mater Chem B 2015;3:3831-3839.

3. Lu S, Wang Y. Single-cell imaging of mechanotransduction in endothelial cells. Prog Mol Biol Transl 2014;126:25-51.

4. Chudakov DM, Matz MV, Lukyanov S, Lukyanov KA. Fluorescent proteins and their applications in imaging living cells and tissues. Physiol Rev 2010;90:1103-1163.
5. Zhang X, Zhang X, Tao L, Chi Z, Xu J, Wei Y. Aggregation induced emission-based fluorescent nanoparticles: Fabrication methodologies and biomedical applications. J Mater Chem B 2014;2: 4398-4414.

6. Li Y, Guo Y, Niu W, Chen M, Xue Y, Ge J, Ma PX, Lei B. Biodegradable multifunctional bioactive glass-based nanocomposites elastomers with controlled biomineralization activity, real-time bioimaging tracking and decreased inflammatory response. ACS Appl Mater Interfaces 2018;10:17722-17731.

7. Ma C, Xie G, Zhang X, Yang L, Li Y, Liu H, Wang K, Wei Y. Biocompatible fluorescent polymers from pegylation of an aggregation-induced emission dye. Dyes Pigments 2017;139:672-680.

8. Li Y, Guo Y, Ge J, Ma PX, Lei B. In situ silica nanoparticles-reinforced biodegradable poly (citrate-siloxane) hybrid elastomers with multifunctional properties for simultaneous bioimaging and bone tissue regeneration. Appl MaterToday 2018;10:153-163.

9. Singh A, Lim CK, Lee YD, Maeng JH, Lee S, Koh J, Kim S. Tuning solid-state fluorescence to the near-infrared: A combinatorial approach to discovering molecular nanoprobes for biomedical imaging. ACS Appl Mater Interfaces 2013;5:8881-8888. 
10. Du $Y$, Ge J, Li $Y$, Ma PX, Lei B. Biomimetic elastomeric, conductive and biodegradable polycitrate-based nanocomposites for guiding myogenic differentiation and skeletal muscle regeneration. Biomaterials 2018;157:40-50.

11. Yang J, Webb AR, Ameer GA. Novel citric acid-based biodegradable elastomers for tissue engineering. Adv Mater 2004;16:511-516.

12. Du $Y, Y u M, G e J, M a P X, C h e n ~ X$, Lei B. Development of a multifunctional platform based on strong, intrinsically photoluminescent and antimicrobial silica-poly(citrates)-based hybrid biodegradable elastomers for bone regeneration. Adv Funct Mater 2015;25: 5016-5029.

13. Du Y, Yu M, Chen X, Ma PX, Lei B. Development of biodegradable poly(citrate)-polyhedral oligomeric silsesquioxanes hybrid elastomers with high mechanical properties and osteogenic differentiation activity. ACS Appl Mater Interfaces 2016;8:3079-3091.

14. Du Y, Xue Y, Ma PX, Chen X, Lei B. Biodegradable, elastomeric, and intrinsically photoluminescent poly(silicon-citrates) with high photostability and biocompatibility for tissue regeneration and bioimaging. Adv Healthc Mater 2016;5:382-392.

15. Zhou L, Xi Y, Yu M, Wang M, Guo Y, Li P, Ma PX, Lei B. Highly antibacterial polypeptide-based amphiphilic copolymers as multifunctional non-viral vectors for enhanced intracellular siRNA delivery and anti-infection. Acta Biomat 2017;58:90-101.

16. Zhao C, Shao L, Lu J, Deng X, Wu Y. Tumor acidity-induced sheddable polyethylenimine-poly (trimethylene carbonate)/DNA/polyethylene glycol-2,3-dimethylmaleicanhydride ternary complex for efficient and safe gene delivery. ACS Appl Mater Interfaces 2016;8: 6400-6410.

17. Alexander JW, Supp DM. Role of arginine and omega-3 fatty acids in wound healing and infection. Adv Wound Care 2014;3:682-690.

18. Chen M, Yin M. Design and development of fluorescent nanostructures for bioimaging. Prog Polym Sci 2014;39:365-395.

19. Yan D, Evans DG. Molecular crystalline materials with tunable luminescent properties: From polymorphs to multi-component solids. Mater Horizons 2014;1:46-57.

20. Wang M, Guo Y, Yu M, Ma PX, Mao C, Lei B. Photoluminescent and biodegradable polycitrate-polyethylene glycol-polyethyleneimine polymers as highly biocompatible and efficient vectors for bioimaging-guided sirna and mirna delivery. Acta Biomater 2017; 54:69-80.

21. Li F, Wang M, Pi G, Lei B. Europium doped monodispersed bioactive glass nanoparticles regulate the osteogenic differentiation of human marrow mesenchymal stem cells. J Biomed Nanotechnol 2018;14:756-764.

22. Yang $Y$, Cui J, Zheng M, Hu C, Tan S, Xiao Y, Yang Q, Liu Y. One-step synthesis of amino-functionalized fluorescent carbon nanoparticles by hydrothermal carbonization of chitosan. Chem Commun 2012;48:380-382.
23. Banerjee S, Veale EB, Phelan CM, Murphy SA, Tocci GM, Gillespie LJ, Frimannsson DO, Kelly JM, Gunnlaugsson T. Recent advances in the development of 1,8-naphthalimide based DNA targeting binders, anticancer and fluorescent cellular imaging agents. Chem Soc Rev 2013;42:1601-1618.

24. Saito K, Chikkade PK, Kanai M, Kuninobu Y. Palladium-catalyzed construction of heteroatom-containing pi-conjugated systems by intramolecular oxidative $\mathrm{c}-\mathrm{h} / \mathrm{c}-\mathrm{h}$ coupling reaction. Chem Eur J 2015;21:8365-8368.

25. Zhou L, Qu X, Guo Y, Wang M, Lei B, Ma PX. A branched glycerol-based copolymer with ultrahigh p65 siRNA delivery efficiency for enhanced cancer therapy. ACS Appl Mater Interfaces 2018;10:4471-4480.

26. Ma Q, Wang Y, Gao X, Ma Z, Song Z. The beneficial effects of l-arginine on cell proliferation and ornithine decarboxylase activity in patients with colorectal adenoma and adenocarcinoma. Ann Oncol 2007;18:Vii82-Vii.

27. $Y u$, Kuo $H$, Huang $L$, Chen $C$, Tain $Y L$, Sheen JM, Tiao $M$, Huang H, Yang K, Ou CY, Hsu TY. L-arginine modulates neonatal lymphocyte proliferation through an interleukin-2 independent pathway. Immunology 2014;143:184-192.

28. Geiger R, Rieckmann JC, Wolf $T$, Basso $C$, Feng $Y$, Fuhrer $T$, Kogadeeva M, Picotti P, Meissner F, Mann M, Zamboni N, Sallusto $F$, Lanzavecchia A. L-arginine modulates $t$ cell metabolism and enhances survival and anti-tumor activity. Cell 2016;167:829.

29. Urano $Y$, Asanuma D, Hama Y, Koyama Y, Barrett T, Kamiya M, Nagano T, Watanabe T, Hasegawa A, Choyke PL, Kobayashi H. Selective molecular imaging of viable cancer cells with ph-activatable fluorescence probes. Nat Med 2009;15:104-109.

30. Patil UV, Ramgir NS, Karmakar N, Bhogale A, Debnath AK, Aswal DK, Gupta SK, Kothari DC. Room temperature ammonia sensor based on copper nanoparticle intercalated polyaniline nanocomposite thin films. Appl Surf Sci 2015;339:69-74.

31. Ma Y, Xu S, Wang S, Wang L. Luminescent molecularly-imprinted polymer nanocomposites for sensitive detection. Trac Trend Anal Chem 2015;67:209-216.

32. Yan J, He W, Li N, Yu M, Du Y, Lei B, Ma PX. Simultaneously targeted imaging cytoplasm and nucleus in living cell by biomolecules capped ultra-small gdof nanocrystals. Biomaterials 2015;59:21-29.

33. Yang $Y$, Feng $Y$, Wang QF, Tang $X$, Zhang G, Liu W. A novel ratiometric fluorescent probe for selective detection of hg2+, cr3+ and al3+ and its bioimaging application in living cells. Sensor Actuat B Chem 2017;253:1055-1062.

34. Kim H, Rao BA, Jeong J, Angupillai S, Choi JS, Nam JO, Lee CS, Son YA. A rhodamine scaffold immobilized onto mesoporous silica as a fluorescent probe for the detection of fe (iii) and applications in bio-imaging and microfluidic chips. Sensor Actuat B Chem 2016; 224:404-412. 